\title{
Approche multi-échelles pour l'étude de l'anisotropie induite par le forgeage en fatigue à grand nombre de cycles ${ }^{\star}$
}

\author{
P.-O. Bouchard, M. Milesi et Y. Chastel \\ CEMEF - Centre de Mise en Forme des Matériaux, Mines ParisTech, France \\ e-mail : Pierre-Olivier.Bouchard@mines-paristech.fr
}

\section{Mots-clés :}

Fatigue à grand nombre de cycles ; approche multi-échelles ; forgeage ; fibrage ; anisotropie
Résumé - Les pièces de forge sont universellement reconnues pour leurs bonnes propriétés mécaniques, notamment en fatigue. L'approche proposée ici consiste à intégrer la simulation du forgeage dans le dimensionnement en fatigue des pièces forgées. Le fibrage et le taux de corroyage sont deux caractéristiques principales du forgeage. À l'aide du logiciel FORGE ${ }^{\circledR}$, le fibrage est calculé tout au long des opérations de mise en forme. Ce fibrage, ainsi que les contraintes résiduelles, sont ensuite introduits dans des outils de dimensionnement pour améliorer la prédiction du calcul de durée de vie en fatigue. Les critères de fatigue actuels, basés sur des modèles isotropes, ne permettent pas de valoriser le sens long de ce fibrage, et par là les pièces de forge, car c'est en général dans ce sens que ces pièces sont le plus sollicitées. Une extension anisotrope du modèle de Papadopoulos est proposée sur la base d'une campagne expérimentale effectuée sur des éprouvettes prélevées à $0^{\circ}, 45^{\circ}$ et $90^{\circ}$ par rapport à la direction de fibrage. Une modélisation à l'échelle micro (DIGIMICRO) permet également de mieux comprendre le rôle des inclusions sur les sollicitations en fatigue. La mise en place d'une chaîne de simulation virtuelle globale à l'échelle d'un composant industriel, associée à des modélisations microstructurales, permet de mieux comprendre et de quantifier le rôle du fibrage sur les propriétés en fatigue à grand nombre de cycles des pièces forgées.

\begin{abstract}
A multiscale finite element approach to deal with high cycle fatigue anisotropy induced by forging. Forged components are recognized for their good mechanical strength and fatigue properties. The methodology presented here consists in improving fatigue analyses of forged components by accounting for the forging simulation stage. Kneading rate and grain flow orientation are two consequences of the forging process. Using the FORGE ${ }^{\circledR}$ software, grain flow orientation is computed all along the forming process simulation. This grain flow orientation, as well as residual stresses, are then used as input data to enhance the fatigue analysis. Usual isotropic fatigue criteria are usually unable to demonstrate the higher fatigue properties of forged components along the grain flow orientation. An anisotropic extension of the Papadopoulos fatigue criterion is defined here. This extension is based on experimental fatigue results obtained on samples extracted at $0^{\circ}, 45^{\circ}$ and $90^{\circ}$ with respect to the grain flow orientation. A numerical modelling is performed at the microscale using the DIGIMICRO software. These simulations give a better understanding on the influence of elongated particles and cluster of particles on high cycle fatigue mechanisms. A virtual simulation chain is set-up to work on real industrial components. This simulation chain, together with microscale numerical modelling demonstrate the positive influence of the grain flow orientation of forged components on high cycle fatigue properties of industrial parts.
\end{abstract}

es pièces forgées ou matricées représentent des éléments clefs pour de très nombreuses structures mécaniques et pour différents secteurs d'activité : automobile, aéronautique, construction mécanique, mines, prothèses biomécaniques, spatial et équipements divers.

Les pièces de forge sont universellement reconnues pour leurs bonnes propriétés mécaniques, notamment en fatigue. Ceci est dû principalement à la microstructure induite par le procédé de forgeage. D'une manière générale, le corroyage (dont le fibrage est l'une des conséquences visibles) a plusieurs effets : amélioration de la compacité du matériau en éliminant des

\footnotetext{
* Cet article est basé sur l'exposé présenté lors des «29e Journées de Printemps 2010 »
} de la SF2M, Paris, 19-20 mai 2010. 
défauts, structure plus fine et plus homogène (structure dendritique brisée) ou encore anisotropie de certaines caractéristiques mécaniques.

À l'heure actuelle, la phase de conception de la gamme de mise en forme, et celle de dimensionnement de pièces industrielles sont souvent dissociées. Le dimensionnement des pièces en service ne prend notamment pas en compte l'histoire thermomécanique et microstructurale des matériaux les constituant. Cela conduit souvent à une estimation trop approximative de leur résistance en condition d'utilisation ou à l'utilisation de coefficients de sécurité importants.

Les codes de simulation numérique des procédés de mise en forme permettent depuis peu de déterminer les propriétés mécaniques d'une pièce après mise en forme, mais également la microstructure du matériau en fonction des conditions thermomécaniques de mise en œuvre.

Les modèles de fatigue (régime oligocyclique ou endurance, sollicitations multiaxiales), et par conséquent les codes de dimensionnement, n'intègrent pas encore toutes ces informations comme données d'entrée. Plusieurs études ont montré que les propriétés en fatigue en régime polycyclique pouvaient provenir préférentiellement du rôle des défauts ou du rôle de la matrice. Les modèles couramment utilisés sont alors de deux types :

- Rôle des défauts : pour des matériaux présentant des tailles de défauts importantes, le critère de Murakami [1] permet d'étudier la tenue à la fatigue à grand nombre de cycles. Basée sur la notion de facteurs d'intensité des contraintes liés aux défauts ou inclusions, et sur l'évolution de la forme et de l'aire de ces défauts projetée sur le plan perpendiculaire à la contrainte principale maximale, cette équation permet de calculer les limites en fatigue d'un matériau soumis à une sollicitation uniaxiale ou biaxiale simple. Des extensions de ce critère ont été proposées dans $[2,3]$.

- Rôle de la matrice : les approches de type plan critique $[4,5]$ sont adaptées à la fatigue multiaxiale pour des pièces présentant des défauts de petite taille. On considère que la rupture n'aura pas lieu si les grains les plus défavorablement orientés présentent une adaptation élastique. Pour le critère de Papadopoulos, la limite de fatigue est représentée par une droite tracée dans l'espace $\left(T_{\Sigma}, \Sigma_{h, \max }\right)$, et caractérisée par l'équation (1) :

$$
T_{\Sigma}+\alpha \Sigma_{h, \max } \leq \beta
$$

où $T_{\Sigma}$ est une cission généralement obtenue par une double maximisation sur différents plans de glissement et pour tout le cycle de chargement. $\Sigma_{h, \max }$ représente le maximum de la contrainte hydrostatique sur tout le cycle de chargement. Les paramètres $\alpha$ et $\beta$ sont des paramètres matériaux déterminés par exemple par des essais de fatigue en traction et en torsion.

Cependant, certaines limitations subsistent. Les approches de type Murakami permettent de prendre en compte la taille et la morphologie des défauts (et donc l'anisotropie) mais sont limitées à des chargements uni-axiaux, voire bi-axiaux. Les approches de type plan critique peuvent être appliquées pour des chargements multiaxiaux, mais ne tiennent pas compte des défauts et de l'anisotropie. Il n'existe donc pas de modèles de fatigue polycyclique permettant de coupler chargements multiaxiaux, défauts et anisotropie.

Dans le cadre du projet ANR OPTIFORGE, la microstructure induite par le forgeage a été prise en compte dans le dimensionnement en fatigue. Ce projet est constitué d'un partenariat industriel et académique complémentaire : un aciériste (Ascometal Creas), deux forgerons (Setforge et Ascoforge), un utilisateur final (PSA), un éditeur de logiciels (Transvalor) ainsi que trois laboratoires (le CEMEF de Mines ParisTech, le LAMPA d'Arts et Métiers ParisTech ainsi que le laboratoire MATEIS de l'INSA de Lyon) et un centre technique (le CETIM). Même si l'état de surface joue un rôle important sur les propriétés en fatigue, seul le rôle de la microstructure induite par le forgeage est étudié ici.

Dans la suite, la méthodologie mise en place dans le cadre du projet OPTIFORGE pour prendre en compte l'histoire thermomécanique du matériau dans le dimensionnement en fatigue est présentée dans un premier temps. Une attention plus particulière est ensuite portée à la modélisation à l'échelle micro et à la prise en compte de l'état inclusionnaire sur les propriétés en fatigue. 


\section{Intégration des caractéristiques des pièces forgées pour le dimensionnement en fatigue}

\section{Cas industriel retenu}

Le cas industriel retenu est un triangle de suspension (PSA) forgé en sept passes, à partir de barreaux fournis par la société Ascometal en METASCO MC (25MnCrSiVB6). Le METASCO MC est un acier bas carbone allié à une forte proportion de manganèse. Il est formé de bainite supérieure et ne possède pas de texture cristallographique marquée. Le fibrage se présente sous la forme de veines riches en inclusions de type MnS, allongées et alignées dans la direction du fibrage.

\section{Campagne expérimentale}

Des essais staircase ont été effectués sur des éprouvettes prélevées à $0^{\circ}, 45^{\circ}$ et $90^{\circ}$ par rapport à la direction du fibrage. Les essais de traction-compression $(R=-1)$ ont été réalisés au LAMPA et les essais en torsion alternée $(R=-1)$ ont été réalisés au laboratoire MATEIS. Les conditions de préparation ont été identiques dans les deux laboratoires, avec un polissage papier 4000 et un traitement thermique de relaxation des contraintes résiduelles. Les résultats du staircase montrent une limite de fatigue plus importante pour un fibrage dans le sens long en traction-compression ( $+15 \%)$ et en torsion alternée $(+6 \%)$. La valeur la plus basse est obtenue en sens travers pour l'essai de traction-compression et à $45^{\circ}$ pour l'essai de torsion. L'anisotropie est plus marquée en traction-compression et une dispersion importante est observée en sens travers $\left(90^{\circ}\right)$ en torsion alternée.

\section{Mécanismes d'endommagement}

Les mécanismes d'endommagement ont été observés au laboratoire MATEIS et au LAMPA. Des essais interrompus ont été réalisés pour les différents sens de prélèvement et pour les deux types de sollicitation. En traction-compression, pour les éprouvettes prélevées dans le sens long, l'amorçage apparaît toujours dans la matrice, à $45^{\circ}$ par rapport à l'axe de sollicitation. Pour les éprouvettes prélevées dans le sens travers, on observe une compétition entre fissures s'amorçant à $45^{\circ}$ dans la matrice, et fissures s'amorçant à $90^{\circ}$ au niveau des amas d'inclusions. En torsion alternée, on observe l'apparition de nombreuses bandes de glissement à $0^{\circ}$ et $90^{\circ}$ dans la matrice. Les fissures $\mathrm{s}^{\prime}$ amorcent dans les zones de forte densité de bandes de glissement, puis se propagent le long des veines d'inclusions.

\section{Forgeage}

Le triangle de suspension est obtenu en sept passes de forgeage. La modélisation de ces sept passes est effectuée avec le logiciel FORGE ${ }^{\circledR}$, particulièrement bien adapté à la modélisation des matériaux en grandes déformations (fig. 1a).

Le fibrage est calculé tout au long des opérations de forgeage à partir du tenseur gradient des déformations. La figure $1 b$ montre le fibrage observé après attaque sur une coupe du triangle forgé, tandis que la figure 1c montre le fibrage prédit par le calcul. Le taux de corroyage ${ }^{1}$ peut également être évalué à partir du taux de déformation calculé au cours du forgeage [6-8].

En fin de simulation de forgeage, des informations sur l'état thermomécanique de la matière (plasticité, contraintes résiduelles), mais aussi sur sa microstructure (orientation du fibrage, taux de corroyage) sont donc disponibles.

\section{Chargement cyclique}

Des essais de fatigue sont réalisés par Ascometal Creas sur le triangle de suspension. Deux extrémités du triangle sont bloquées alors que la troisième est soumise à un chargement cyclique, comme indiqué en figure 2. Au niveau expérimental, l'effort correspondant à la limite d'endurance est de $11083 \mathrm{~N}$ avec un écart-type de $204 \mathrm{~N}$. La rupture s'amorce dans le bras sollicité, à une distance d'environ $100 \mathrm{~mm}$ de l'axe de chargement.

\section{Prise en compte de la microstructure induite par le forgeage dans le dimensionnement en fatigue}

Deux approches ont été développées pour intégrer les nouvelles informations provenant de la simulation du forgeage (fibrage,

1 Rapport de la section de départ du lingot à la section de l'ébauche obtenue. Le taux de corroyage indique l'importance du travail de déformation effectué. 


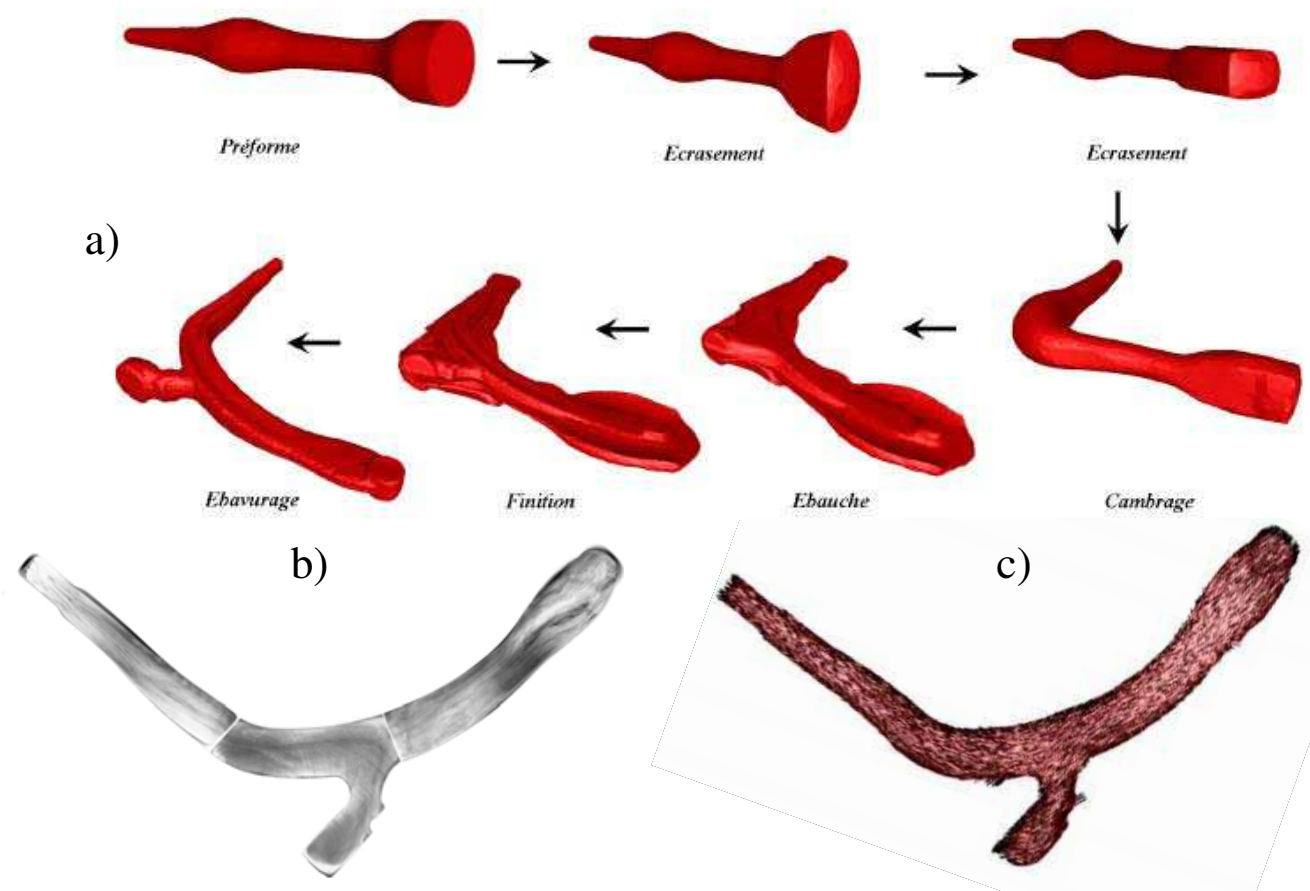

Fig. 1. (a) Modélisation du forgeage en sept passes du triangle de suspension, (b) fibrage expérimental et (c) fibrage calculé.

Fig. 1. (a) Numerical modelling of the wishbone suspension with seven forging operations, (b) experimental grain flow orientation and (c) computed grain flow orientation.

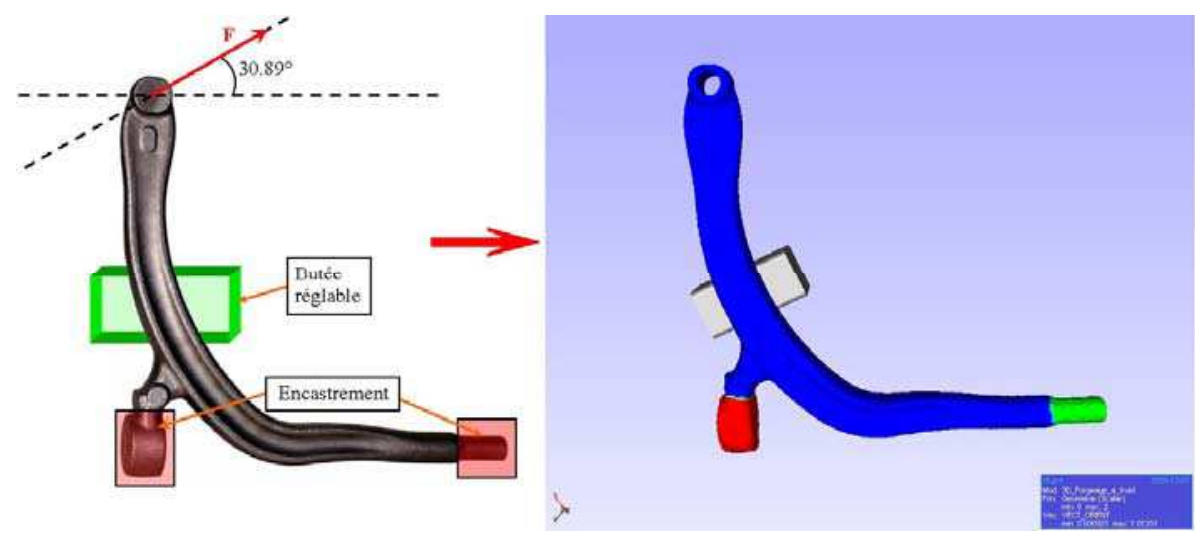

Fig. 2. Chargement cyclique appliqué sur le triangle de suspension.

Fig. 2. Cyclic loading applied to the wishbone suspension.

taux de corroyage) dans le dimensionnement en fatigue.

Le LAMPA a étendu le critère de Morel [9] pour prendre en compte les deux mécanismes d'amorçage observés lors de la campagne expérimentale (amorçage dans la matrice ou amorçage dans les chapelets d'inclusions). Le modèle probabiliste anisotrope ainsi développé est présenté dans [10].

Dans la suite, une approche basée sur des calculs à l'échelle micro, prenant en compte explicitement la présence des chapelets d'inclusions, est présentée.

\section{Modélisation à l'échelle micro}

L'anisotropie observée dans le METASCO MC provient très majoritairement de l'orientation des chapelets d'inclusion, et la matrice semble présenter des propriétés isotropes.

Nous faisons ici l'hypothèse que le modèle de Dang Van, ou de Papadopoulos, peut 


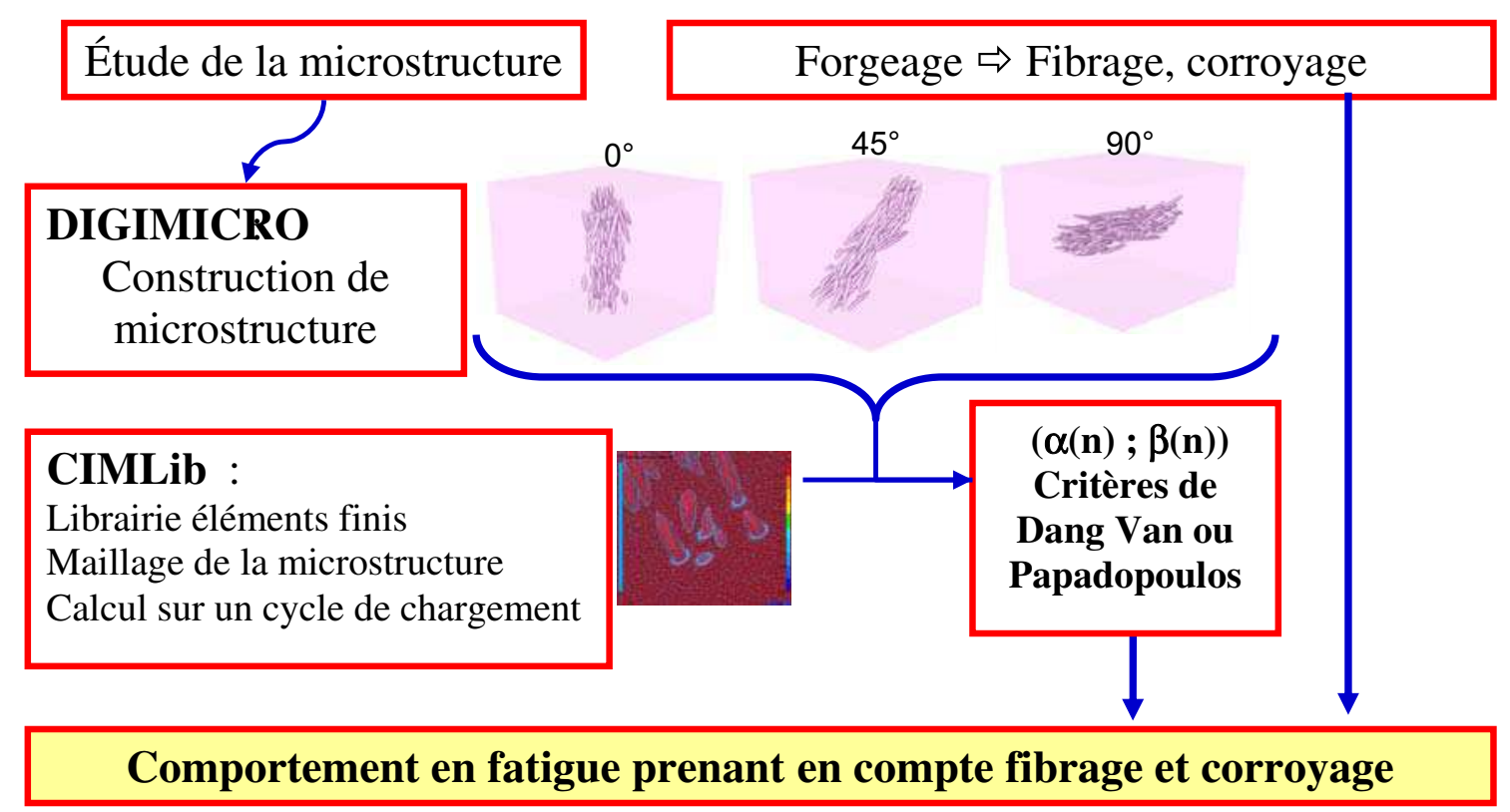

Fig. 3. Approche micro-macro pour l'étude de l'influence de la mise en forme sur les propriétés en fatigue.

Fig. 3. Multiscale methodology to study the influence of the forming process on fatigue properties.

être utilisé dans la matrice à l'échelle d'un volume élémentaire représentatif (VER), et nous souhaitons mettre en évidence l'anisotropie de notre matériau au travers de l'orientation, de la morphologie et de la compacité des chapelets d'inclusions. Pour cela, la méthodologie présentée sur la figure 3 est mise en place. L'objectif est de déduire des calculs micro des valeurs des paramètres $\alpha$ et $\beta$ qui dépendent de l'orientation du fibrage et du taux de corroyage.

\section{Étude de la microstructure}

Pour obtenir les données microstructurales, le triangle de suspension a été découpé en plusieurs tronçons (fig. 4a). Des analyses MEB, EDS et EBSD ont été réalisées sur des coupes longitudinales et transverses. L'état inclusionnaire est étudié afin de définir des statistiques géométriques en termes de : longueur et largeur de chapelets d'inclusions, orientation et morphologie des inclusions. La figure $4 \mathrm{~b}$ donne un exemple des statistiques ainsi générées et qui pourront être utilisées pour la construction des VER.

Il est notamment intéressant d'observer une diminution de la largeur des chapelets $\mathrm{d}^{\prime}$ inclusions lorsque le taux de corroyage augmente. La largeur des chapelets d'inclusions, observée sur le barreau initial ainsi que dans trois sections du triangle de suspension présentant des taux de corroyage différents, est tracée en figure 5 .

\section{DIGIMICRO : construction de la microstructure numérique}

Les statistiques géométriques ainsi déterminées sont utilisées pour générer des VER à l'aide du logiciel DIGIMICRO développé au CEMEF (fig. 6).

\section{CIMLib : maillage éléments finis}

La librairie CIMLib est utilisée pour réaliser les maillages de ces VER. Il s'agit d'une bibliothèque éléments finis développées en $\mathrm{C}++$ qui possède notamment un mailleur adaptatif anisotrope particulièrement performant pour mailler de telles microstructures [11]. L'utilisation de fonctions level-set permet de localiser les interfaces inclusionsmatrice dans le VER. Le mailleur adaptatif génère alors un maillage en raffinant de manière anisotrope les éléments au voisinage des interfaces : éléments allongés le long des inclusions et très raffinés perpendiculairement à la surface des inclusions (fig. 7). Un tel raffinement anisotrope permet notamment de mieux capter les gradients des champs mécaniques aux interfaces inclusions-matrice.

\section{Données mécaniques}

Les données mécaniques élastiques sont obtenues de la manière suivante. Le comportement du matériau (matrice + inclusions) 


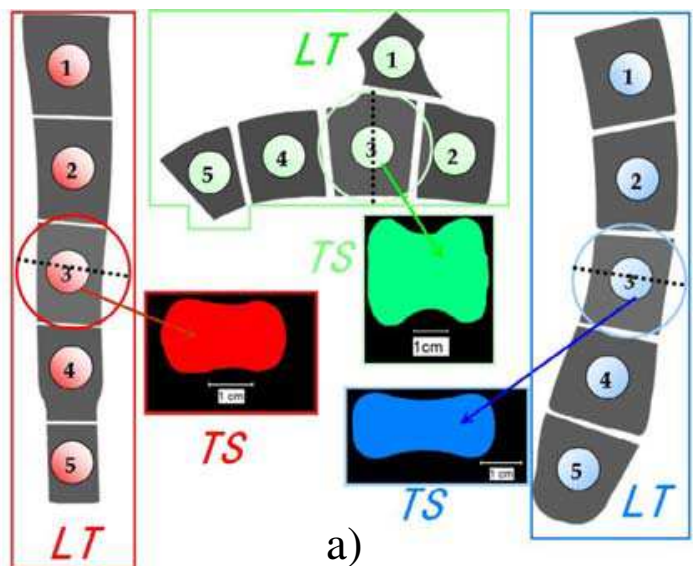

a)
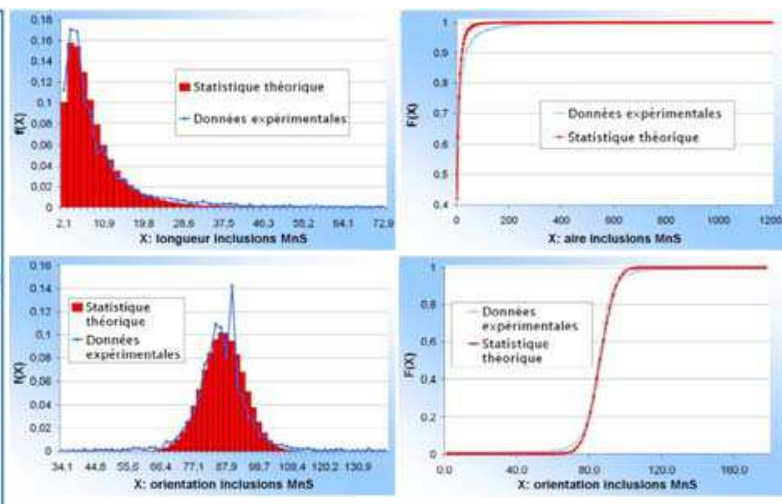

b)

Fig. 4. (a) Découpe du triangle en plusieurs morceaux et (b) analyse statistique de l'état inclusionnaire.

Fig. 4. (a) Wishbone suspension cut in several parts and (b) statistical analysis of particles distribution, morphologies and orientations.

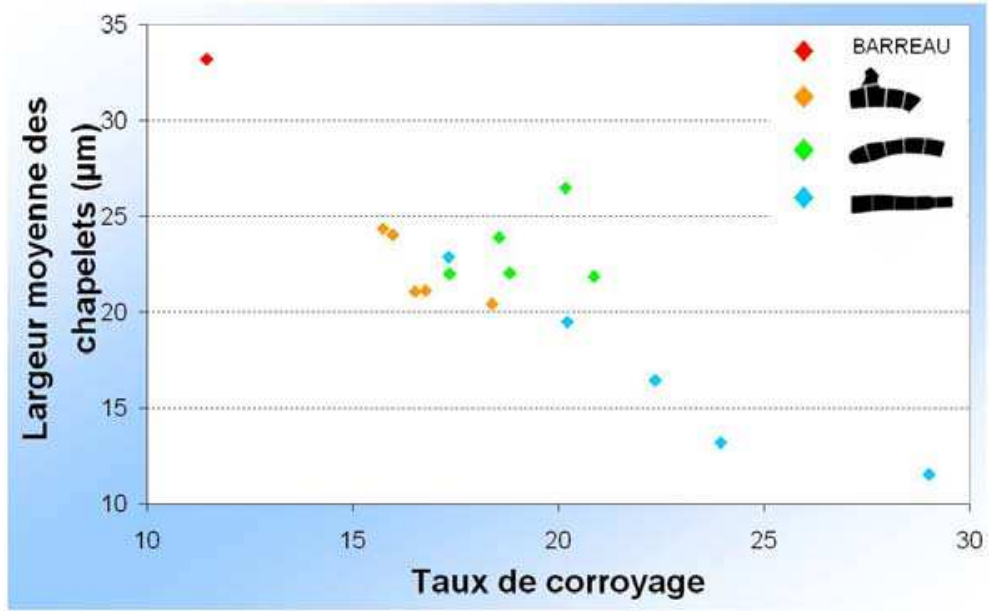

Fig. 5. Évolution de la largeur des chapelets d'inclusions en fonction du taux de corroyage. Fig. 5. Width of cluster of particles with respect to the kneading rate.

est obtenu à partir d'essais monotones. Le comportement des inclusions est obtenu par nanodureté. Un grand nombre de mesures est effectué de manière à définir une statistique sur les modules d'Young. Le comportement mécanique de la matrice est enfin obtenu à partir des deux données précédentes et en tenant compte du taux inclusionnaire dans le METASCO MC.

Pour les propriétés en fatigue, les coefficients $\alpha$ et $\beta$ de la matrice sont déterminés à partir d'essais de traction-compression et de torsion alternée sur des éprouvettes présentant un fibrage à $0^{\circ}$. Nous considérons donc que les propriétés en fatigue de la matrice sont isotropes en formulant les deux hypothèses issues des observations expérimentales suivantes :

- Pour un fibrage à $0^{\circ}$, les chapelets $d$ 'inclusions ne semblent pas influencer l'amorçage des fissures qui se situe dans la matrice.
- La limite de fatigue en torsion alternée est peu dépendante de l'orientation du fibrage.

Le jeu de paramètres $\alpha$ et $\beta$ correspond ainsi à la matrice considérée comme isotrope. Par la suite, l'anisotropie est considérée comme provenant de la morphologie et de l'orientation des chapelets d'inclusions.

\section{Mise en évidence de l'anisotropie à l'échelle micro}

Chaque VER généré est maillé et un calcul élément fini est effectué pour un cycle de chargement. Une contrainte de tractioncompression est appliquée au niveau du VER et le critère de Papadopoulos est utilisé dans la matrice avec les paramètres $\alpha$ et $\beta$ identifiés précédemment (fig. 8a). Nous faisons donc ici l'hypothèse forte que le critère de Papadopoulos peut être utilisé dans 


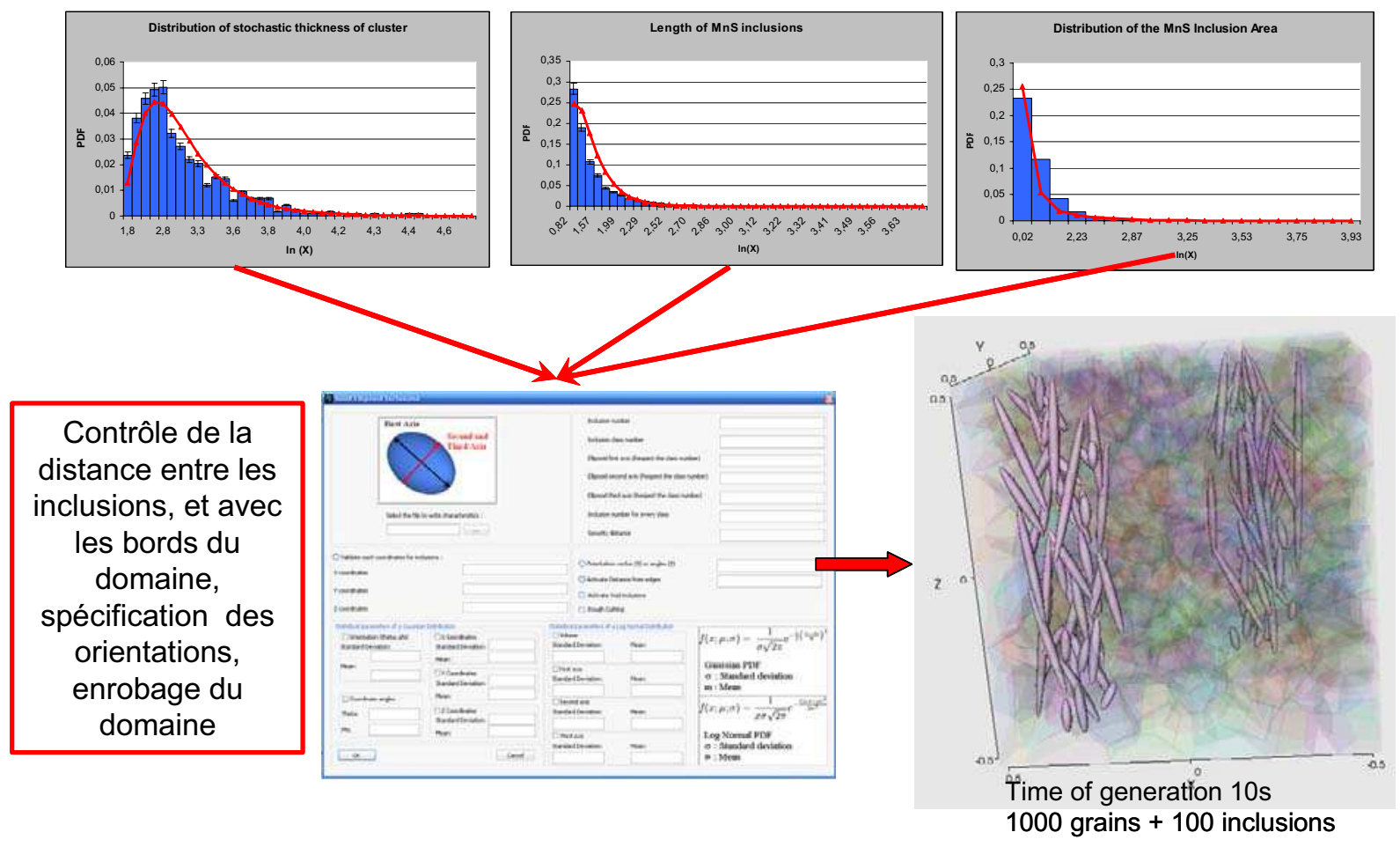

Fig. 6. Construction de VER géométriques sur la base des données statistiques obtenues par analyse d'image. Fig. 6. Construction of a geometrical REV based on statistical data from image analyses.

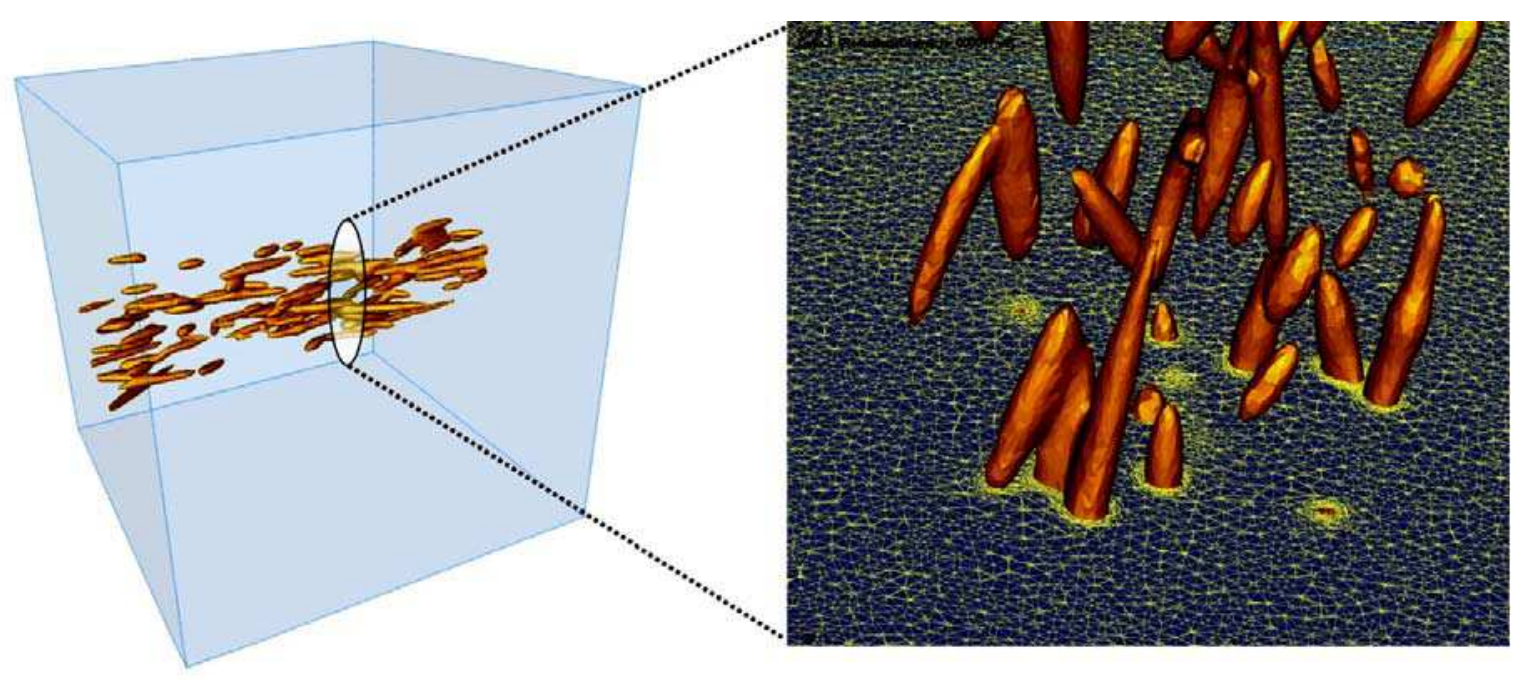

Fig. 7. Exemple de maillage d'un VER contenant un chapelet d'inclusions, avec raffinement anisotrope adaptatif au voisinage des inclusions.

Fig. 7. Example of a REV mesh containing a cluster of elongated particles; anisotropic mesh refinement is performed at inclusions-matrix interfaces.

la matrice à cette échelle. Plusieurs calculs ont été effectués en faisant varier l'orientation du fibrage ou encore la morphologie des chapelets d'inclusions tels qu'observés dans chacune des trois zones du triangle.

Sur la figure $8 b$, l'évolution de l'endurance limite pour chaque VER ainsi généré est tracée. Chaque point indiqué est issu de plusieurs calculs qui intègrent le caractère statistique des observations géométriques réalisées. On observe tout d'abord la décrois- sance de la limite d'endurance lorsque l'on passe $d^{\prime}$ un fibrage à $0^{\circ}$ à un fibrage à $45^{\circ}$ puis à $90^{\circ}$. Le résultat numérique obtenu pour le VER correspondant à celui du barreau avant forgeage est cohérent avec les résultats obtenus expérimentalement sur ce même barreau (trait pointillé). Une légère augmentation de la limite de fatigue avec le taux de corroyage est également observée. Cette augmentation est plus marquée pour un fibrage à $90^{\circ}$ que pour un fibrage à $0^{\circ}$. En effet, 


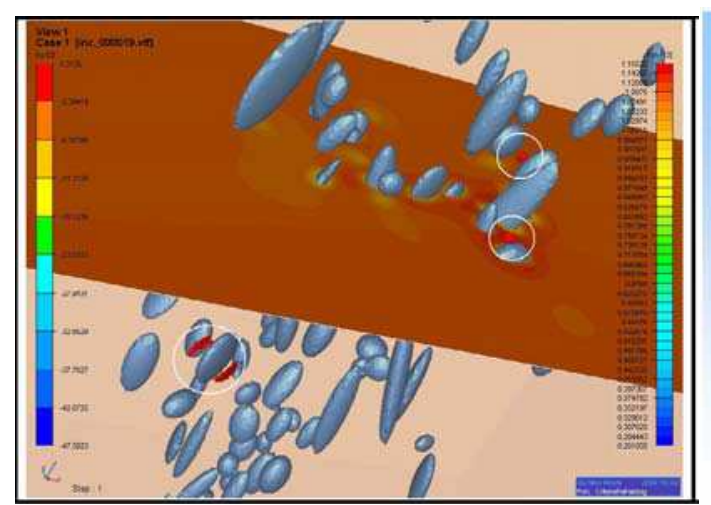

a)

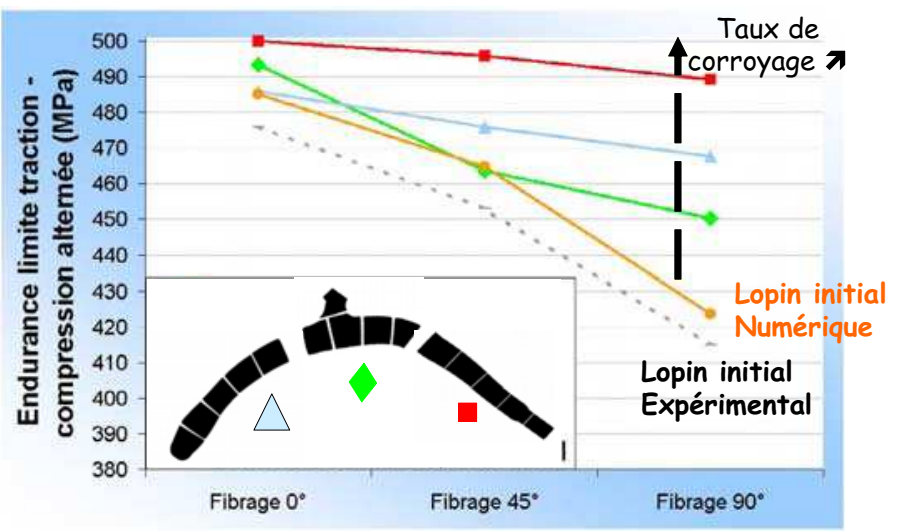

b)

Fig. 8. (a) Prédiction du critère de Papadopoulos à l'échelle du VER et (b) mise en évidence de l'anisotropie des propriétés en fatigue sur la base des calculs micro.

Fig. 8. (a) Values of the Papadopoulos criterion at the REV scale and (b) fatigue properties anisotropy based on microscale computations.

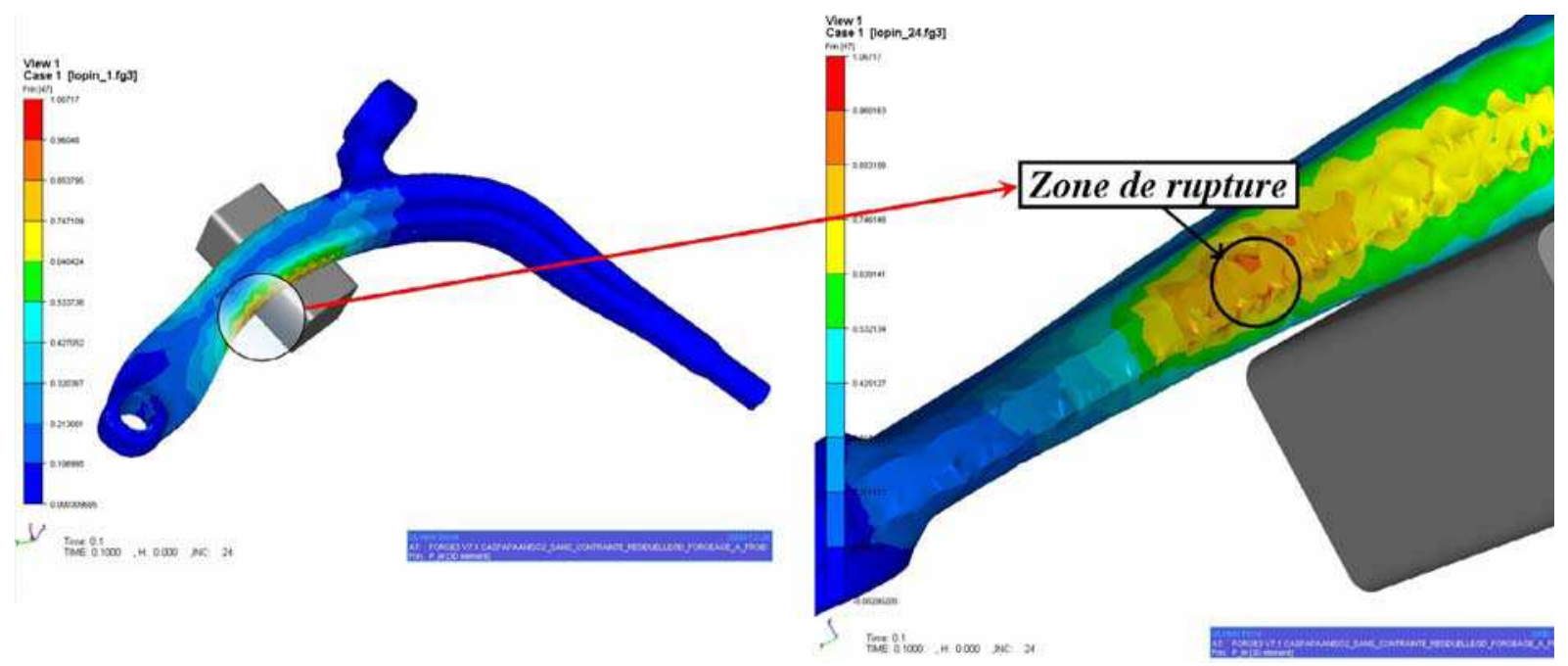

Fig. 9. Localisation de la rupture sur le triangle avec le modèle de Papadopoulos anisotrope.

Fig. 9. Fracture location on the wishbone suspension obtained with the anisotropic Papadopoulos criterion.

il a été montré qu'à partir d'un certain taux de corroyage limite, il n'y avait plus d'augmentation de la limite d'endurance avec le taux de corroyage. Le taux de corroyage initial du barreau étant déjà relativement élevé, il n'a pas une grande influence sur nos résultats.

\section{Prise en compte de l'anisotropie à l'échelle macro}

Les calculs réalisés sur VER permettent de définir des paramètres $\alpha$ et $\beta$ en fonction de l'orientation du fibrage et du taux de corroyage. Un cycle de chargement est réalisé sur le triangle avec les conditions aux limites définies sur la figure 2 , et le critère de Papadopoulos est utilisé avec les paramètres $\alpha$ et $\beta$ anisotropes. La prédiction du critère est en bonne adéquation avec les résultats expérimentaux (fig. 9), que ce soit au niveau de la localisation de la rupture $(107 \mathrm{~mm}$ de l'axe de chargement) ou de l'effort conduisant à la rupture par fatigue. Au niveau expérimental, l'effort correspondant à la limite d'endurance est de $11083 \mathrm{~N}$ avec un écarttype de $204 \mathrm{~N}$; et pour la simulation numérique, cet effort est de $11279,6 \mathrm{~N}$ pour un écart-type de 276,2 N.

\section{Conclusion}

Dans cet article, une méthodologie multiéchelle permettant d'évaluer l'anisotropie des propriétés en fatigue sur la base de calculs à l'échelle d'un VER a été présentée. L'anisotropie en fatigue du METASCO MC provenant majoritairement de l'état inclusionnaire, la modélisation d'un VER contenant des chapelets d'inclusions a permis 
de retrouver l'effet bénéfique du fibrage et du taux de corroyage sur les propriétés en fatigue.

Si les résultats obtenus ici sont tout à fait concluants, il convient cependant de préciser certaines limitations qu'il faudra aborder par la suite :

- Lors du refroidissement du matériau, certaines contraintes résiduelles peuvent apparaître aux interfaces inclusions/matrice. Ces contraintes résiduelles devront être évaluées et intégrées au modèle pour améliorer sa prédictivité.

- L'approche présentée ici ne tient pas compte de l'état de surface, mais uniquement de l'état de la matière via le fibrage et le taux de corroyage. Cet état de surface jouant un rôle important sur les propriétés en fatigue, il sera important de l'intégrer dans cette démarche.

- Enfin, cette méthodologie convient aux matériaux pour lesquels l'anisotropie provient des inclusions et non de la matrice.

Quoi qu'il en soit, la représentation de l'état inclusionnaire à cette échelle revêt un intérêt majeur pour la compréhension des mécanismes de fatigue ou encore d'endommagement ductile pour lesquels l'état inclusionnaire joue un rôle prépondérant.

\section{Références}

[1] Y. Murakami, M. Endo, Int. J. Fatigue 16 (1994) 163-182

[2] N. Caillet, Prise en compte des spécificités des pièces forgées en fatigue illimitée, Ph.D. thesis, École Nationale Supérieure des Mines de Paris, 2007

[3] Y. Chastel, N. Caillet, P.O. Bouchard, J. Mat. Process. Tech. 177 (2006) 202-205

[4] K. Dang Van, B. Griveau, O. Message, Mech. Engrg. Pub., EGF 3 (1989) 479-496

[5] I.V. Papadopoulos, V.P. Panoskaltsis, Engrg. Fract. Mech. 55 (1996) 513-528

[6] M. Milesi, Impact des caractéristiques microstructurales des pièces forgées sur leur tenue en fatigue à grand nombre de cycles, Ph.D. thesis, École Nationale Supérieure des Mines de Paris, 2009

[7] M. Milesi, Y. Chastel, M. Bernacki, R. Loge, P.O. Bouchard, Comp. Meth. Mater. Sci. 7 (2007) 383-388

[8] M. Milesi, Y. Chastel, E. Hachem, M. Bernacki, R.E. Logé, P.O. Bouchard, Mater. Sci. Eng. A 527 (2010) 4654-4663

[9] F. Morel, Int. J. Fatigue 22 (2000) 101-119

[10] E. Pessard, Comportement anisotrope en fatigue des composants mécaniques forges, Ph.D. thesis, École Nationale Supérieure des Arts et Métiers, 2009

[11] M. Bernacki, Y. Chastel, T. Coupez, R.E. Logé, Scripta Mater. 58 (2008) 1129-1132 